\title{
Prevalence of Influenza A(H1N1)pdm09 Virus Resistant to Oseltamivir in Shiraz, Iran, During 2012 - 2013
}

\author{
Nastaran Khodadad ${ }^{1}$; Afagh Moattari ${ }^{1,{ }^{*}}$; Mahmoud Shamsi Shahr Abadi ${ }^{2}$; Mohammad \\ Rahim Kadivar ${ }^{3}$; Jamal Sarvari ${ }^{1}$; Forough Tavakoli ${ }^{1}$; Neda Pirbonyeh ${ }^{1}$; Amir Emami ${ }^{1}$ \\ ${ }^{1}$ Department of Bacteriology and Virology, Shiraz University of Medical Sciences, Shiraz, IR Iran \\ ${ }^{2}$ Department of Virology, Tehran University of Medical Sciences, Tehran, IR Iran \\ ${ }^{3}$ Department of Pediatrics, Namazi Hospital, Shiraz University of Medical Sciences, Shiraz, IR Iran \\ *Corresponding author:Afagh Moattari, Department of Bacteriology and Virology, Shiraz University of Medical Sciences, Shiraz, IR Iran. Tel/Fax:+98-7132304356, E-mail: moattaria@yahoo.com
}

Received: September 24, 2014; Revised: November 16, 2014; Accepted: January 21, 2015

\begin{abstract}
Background: Oseltamivir has been used as a drug of choice for the prophylaxis and treatment of human influenza A(H1N1)pdm09 infection across the world. However, the most frequently identified oseltamivir resistant virus, influenza A(H1N1)pdm09, exhibit the H275Y substitution in NA gene.

Objectives: This study aimed to determine the prevalence and phylogenetic relationships of oseltamivir resistance in influenza A(H1N1) pdm09 viruses isolated in Shiraz, Iran.

Patients and Methods: Throat swab samples were collected from 200 patients with influenza-like disease from December 2012 until February 2013. A total of 77 influenza A(H1N1)pdm09 positive strains were identified by real-time polymerase chain reaction (PCR). Oseltamivir resistance was detected using quantal assay and nested-PCR method. The NA gene sequencing was conducted to detect oseltamivir-resistant mutants and establish the phylogeny of the prevalent influenza variants.

Results: Our results revealed that $\mathrm{A}(\mathrm{H} 1 \mathrm{N1}) \mathrm{pdm09}$ viruses present in these samples were susceptible to oseltamivir, and contained 5 site specific mutations (V13G, V106I, V241I, N248D, and N369K) in NA gene. These mutations correlated with increasing expression and enzymatic activity of NA protein in the influenza A(H1N1)pdm09 viruses, which were closely related to a main influenza A(H1N1)pdm09 cluster isolated around the world.

Conclusions: A(H1N1)pdm09 viruses, identified in this study in Shiraz, Iran, contained 5 site specific mutations and were susceptible to oseltamivir.
\end{abstract}

Keywords: Influenza A(H1N1) Virus; Oseltamivir; Iran

\section{Background}

The seasonal influenza $\mathrm{A}(\mathrm{H} 1 \mathrm{~N} 1)$ virus is responsible for respiratory tract infections in humans and its morbidity and mortality are increasing (1-3). During pandemic 2009, a novel influenza A virus was emerged in North America and Mexico and quickly spread to many countries across the world. Subsequently, the world health organization (WHO) enhanced phase 6 for the universal pandemic alert level urging prevention and treatment strategies through vaccination and administration of anti-influenza drugs such as adamantanes and neuraminidase inhibitors $(1,2,4)$. Adamantanes block M2 ion channel protein and oseltamivir and zanamivir, which are neuraminidase inhibitors (NAIs) and block the activity of viral neuraminidase (NA). NAIs specifically inhibit viral replication by preventing virus release and allowing virus accumulation in cells (5-7).

The quick emergence and spread of resistance to oseltamivir were observed in the 2009 pandemic influenza (A(H1N1)pdm09) (4, 8). Resistance to oseltamivir can be identified by genotypic and phenotypic assays (9). Most A(H1N1)pdm09 viruses have a specific histidine to tyrosine substitution at position 275 (H275Y) of neuraminidase protein, which is responsible for resistance to oseltamivir $(3,4,7,8,10)$. Recently, another amino acid substitution in the NA (serine to asparagines, S247N) of $\mathrm{A}(\mathrm{H} 1 \mathrm{N1})$ pdmo9 viruses has been identified, that designates a low-level of oseltamivir-resistance $(7,10,11)$. Until February 2009, data reported from Argentina indicated that $97 \%$ of the examined European specimens were resistant to oseltamivir, which were sensitive to amantadine, rimantadine, and zanamivir (7).

\section{Objectives}

The goals of this study were to determine the prevalence of oseltamivir resistant influenza A(H1N1)pdm09 viruses in Shiraz, Iran from December 2012 until February 2013, and compare the phylogenetic relationships between these viruses from Iran and other countries.

Copyright (C) 2015, Ahvaz Jundishapur University of Medical Sciences. This is an open-access article distributed under the terms of the Creative Commons Attribution-NonCommercial 4.0 International License (http://creativecommons.org/licenses/by-nc/4.0/) which permits copy and redistribute the material just in noncommercial usages, provided the original work is properly cited. 


\section{Patients and Methods}

\subsection{Sample Collection}

Throat swab samples from 200 patients (44.5\% from females and 55.5\% from males), with influenza-like illnesses were collected in Shiraz hospitals from December 2012 until February 2013. The patients aged from 2.5 months to 70 years, with the mean age of 10.01 years. The study was approved by the ethics committee of Shiraz university of medical sciences.

\subsection{Cells and Virus Isolation}

Madin-Darby canine kidney cell was grown in DMEM medium (Sigma-Aldrich, Germany) supplemented with $10 \%$ fetal bovine serum (GIBCO, Australia), containing 100 $\mu \mathrm{g} / \mathrm{mL}$ penicillin and $100 \mu \mathrm{g} / \mathrm{mL}$ streptomycin. The isolation of influenza virus in Madin-Darby canine kidney cells was carried out using standard procedure described previously. After observing the cytopathic effect, the hemagglutinin assay (HA) test was performed using guinea pig erythrocytes (12).

\subsection{Oseltamivir Drug Susceptibility Testing}

Oseltamivir cytotoxicity was determined by growing uninfected cells in the absence or presence of various concentrations of oseltamivir for 1, 2,3, and 4 days (Roche, UK). The cytotoxicity test was carried out using Microculture Tetrazolium assay (MTT) (Sigma, Germany) (13). MDCK cell infected with the isolated viral strains were incubated at $34^{\circ} \mathrm{C}$, for 1 hour. The supernates were then replaced with medium containing various concentrations of oseltamivir carboxylate, and incubated at $34^{\circ} \mathrm{C}$ for 72 hours. The oseltamivir susceptibility was determined using Spearman-Karber method of measuring 50\% tissue culture infective dose $\left(\operatorname{TCID}_{50}\right)(14)$.

\subsection{RNA Extraction}

RNA extraction was carried out using Roche high pure viral RNA extraction kit (Roche, Germany) according to the manufacturer's instructions. Extracted RNAs were kept in $-80^{\circ} \mathrm{C}$ until further processing.

\subsection{Real-Time Polymerase Chain Reaction}

Real-time PCR was carried out using SuperScript III Platinum one-step quantitative RT-PCR kit (Invitrogen, USA). Real-time runs were performed on the Corbett 6000 Rotor-Gene system. The reaction comprised $4 \mu \mathrm{L}$ of extracted RNA combined with $16 \mu \mathrm{L}$ of master mix, including $2 \mathrm{X}$ reaction mix; SuperScript III RT/Platinum Taq Mix; $5.4 \mu \mathrm{L}$ RNase/DNase-Free water; and $0.4 \mu \mathrm{L}$ of each primer and probe with the $40 \mu \mathrm{L}$ and $10 \mu \mathrm{L}$ concentrations, respectively.

Each sample of RNA was tested by separate primer/ probe sets for the detection of influenza universal swine
(swFLuA), Swine H1, and RNase P. According to the CDC real-time PCR protocol (2009), the cycling conditions included a 30 minutes RT step at $50^{\circ} \mathrm{C}$, followed by enzyme inactivation at $95^{\circ} \mathrm{C}$ for 2 minutes. The PCR step comprised 45 cycles at $95^{\circ} \mathrm{C}$ for 15 seconds, $55^{\circ} \mathrm{C}$ for 30 seconds, and $72^{\circ} \mathrm{C}$ for 30 seconds. Data acquisition and analyses of the real-time PCR assay were accomplished using the Rotor-Gene data analysis Software, Version 6.0A.

\subsection{Real Time Polymerase Chain Reaction and Se- quencing}

RNA was converted to cDNA using 2-steps RT-PCR kit (Vivantis, Malaysia). NA gene was amplified and RT-PCR products were purified employing RT-PCR purification kit (Bioneer, Korea), and then sequenced applying Nested-PCR internal primers (SinaClon, Iran) (15). Chromatogram of our sequences were aligned with the consensus sequence of $\mathrm{A}(\mathrm{H} 1 \mathrm{~N} 1) \mathrm{pdm} 09$ derived from sequences recorded in NCBI (national center for biotechnology information) by Chromas software and resistance mutations were studied using CLC Sequence Viewer 6 software.

Neuraminidase sequences obtained were deposited at GenBank database (USA) under accession numbers: KJ755333, KJ755334, KJ755335, KJ755336, KJ789325, KJ789326, KJ789327, KJ789328, KJ789329, and KJ789330. The whole NA gene alignment was performed using the ClustalW2 program.

The phylogenetic tree of the NA gene was drawn using the neighbor-joining method and analyzed by the bootstrap consensus tree method with 1000 replicates. The evolutionary distances were calculated using the p-distance method. The analysis involved 36 NA gene nucleotide sequences. Evolutionary analyses were performed in the MEGA program (version 6.0) (16). The tree was rooted with a reference strain A/California/4/2009 NA sequence (GenBank accession number: FJ966084), representing the viral sequences obtained at the beginning of the pandemic.

\section{Results}

\subsection{Virus Isolation}

The mutations linked to the drug-resistant variants of influenza viruses were studied to determine the current status of oseltamivir resistant $\mathrm{A}(\mathrm{H} 1 \mathrm{N1}) \mathrm{pdm09}$ viruses in Shiraz, Iran. Across 200 specimens, 77 (38.5\%) isolates were identified as $\mathrm{A}(\mathrm{H} 1 \mathrm{N1}) \mathrm{pdm} 09$ viruses using RT-PCR method, hemagglutinin assay, and NA-specific Nested PCR and genetic sequencing. The genetic sequencing then revealed that 10 of the $\mathrm{A}(\mathrm{H} 1 \mathrm{~N} 1)$ pdmog viruses isolated during 2012 - 2013 were wild-type (H275) in the NA gene. The symptoms observed in most patients studied, were fever, chills, muscle aches, headache, cough, fatigue, and less frequently sore throat, pharyngitis, nasal congestion, digestive disturbance, and otitis. 
4.2. Phylogenetic Analysis of the Neuraminidase Gene

The sequences and phylogenetic relationships of the 10 influenza viruses isolated from Shiraz, Iran during 2012 and 2013 demonstrated that they belonged to A, B, and C clusters based on their NA gene. In this phylogeny, most isolates located within A and B clusters with V13G, V106I, V241I, N248D, and N369K mutations in the NA gene. We could not detect any oseltamivir-resistant H275Y mutation in NA gene in our samples. This also indicated a close phylogenetic relationship between A(H1N1)pdmo9 viruses detected from Tehran and Shiraz (Figure 1). Phylogenetic analysis of different A(H1N1)pdm09 viruses in 2013 was also consistent with isolation of A(H1N1)pdm09 viruses isolated around the world during 2013 (17). These sequences of NA gene, including those of our isolates formed a single cluster (Figure 1).

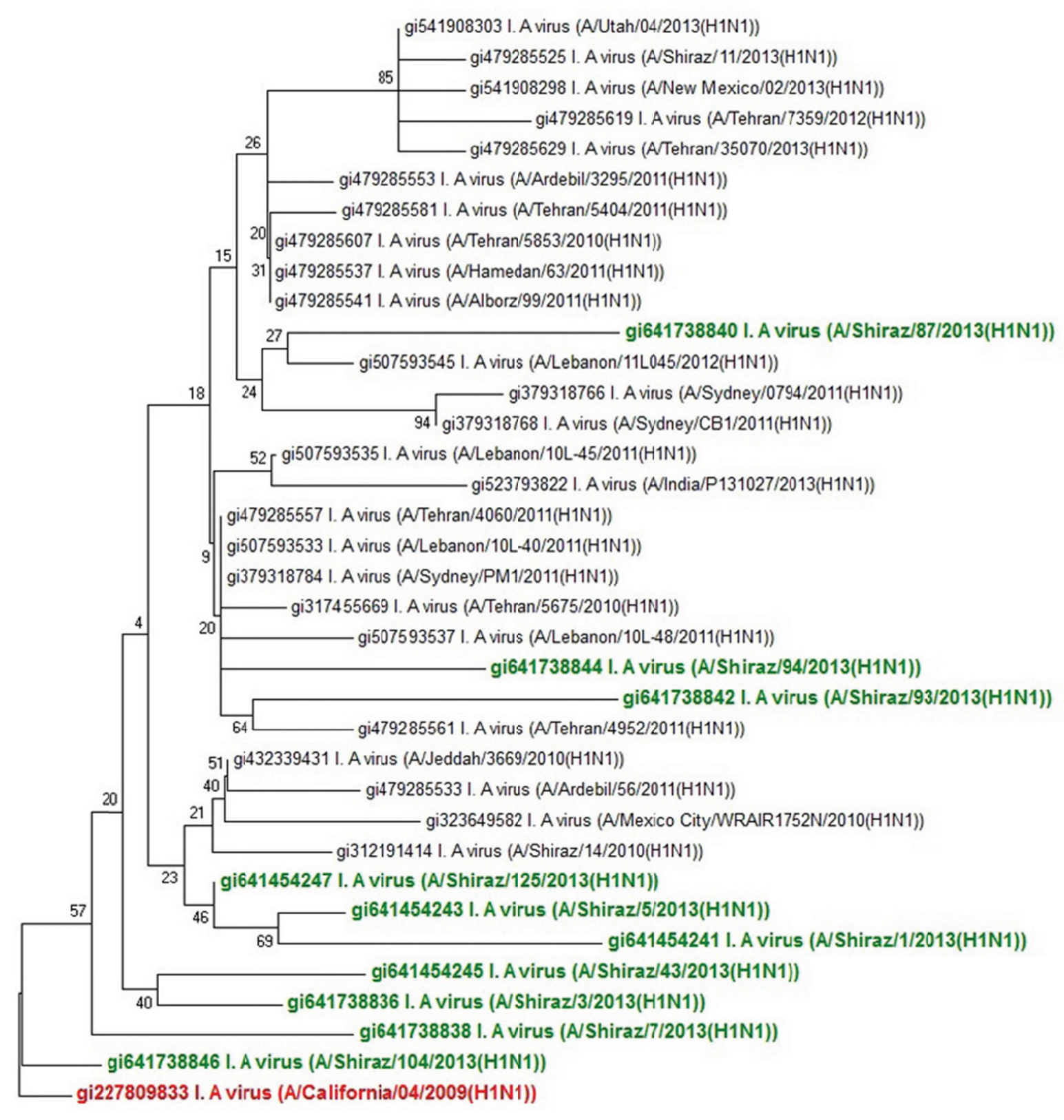

0.005

Figure 1. Phylogenetic Analysis of Neuraminidase Gene Nucleotide Sequences From Influenza A(H1N1)pdm09 Viruses Isolated in Shiraz, Iran, From 2012 to 2013 
(A comparison between nucleotide sequences of A(H1N1)pdm09 viruses isolated in Shiraz, Iran in 2013 and sequences of $\mathrm{A}(\mathrm{H} 1 \mathrm{~N} 1) \mathrm{pdm} 09$ viruses from Iran and other parts of the world, indicated by their GI numbers, also bootstrap values are shown on the branches adjacent to the tree nodes. The strains from Shiraz and a reference strain are shown in bold green and bold red respectively).

\subsection{Genotypic and Phenotypic Antiviral Drug-Sus- ceptibility}

All our isolates were sensitive to oseltamivir in culture and this was evidenced by the lack of H275Y and S247N mutations and 9 substitutions (V116, I117, E119, Q136, K150, D151, D199, I223 and N295) in the NA gene as indicators of NAI resistance demonstrated by CLC Sequence Viewer 6 software $(18,19)$. Statistical analysis using t test showed significant differences in the frequency of oseltamivir resistance between various concentrations of the drug in 3 replicates $(\mathrm{P}<0.001)$ (Table 1$)$. All 10 oseltamivir sensitive isolates had $\mathrm{TCID}_{50}$ values between 0.49 and 5.32 (Table 1). The analysis of full-length NA sequences obtained from A(H1N1)pdm09 viruses collected during December 2012 until February 2013 in Shiraz, Iran revealed close relationship between these viruses evidenced by $97 \%$ 99.78\% similarity among neuraminidase nucleotides. A comparison of these samples' sequences showed 5 additional substitutions (V13G, V106I, V241I, N248D, and N369K) in NA protein (Table 2). These mutations were typically present in many of the 2013 sequences and were not associated with resistance pattern.

Table 1. The Oseltamivir Drug Susceptibility Testing of Influenza A(H1N1)pdm09 Viruses Isolated in Shiraz, Iran

\begin{tabular}{lccc}
\hline Oseltamivir Concentration, $\mathbf{m g} / \mathbf{m L}$ & Mean of TCID $(\mathbf{5 0})$ Log10 Virus Yield & Effect (S or R) & P Value \\
\hline $\mathbf{0}$ & $6 \pm 0$ & - & - \\
$\mathbf{0 . 5}$ & $0.49 \pm 0.49$ & $\mathrm{~S}$ & $<0.001$ \\
$\mathbf{0 . 2 5}$ & $0.92 \pm 0.51$ & $\mathrm{~S}$ & $<0.001$ \\
$\mathbf{0 . 1 2 5}$ & $1.29 \pm 0.50$ & $\mathrm{~S}$ & $<0.001$ \\
$\mathbf{0 . 0 6 2 5}$ & $1.54 \pm 0.52$ & $\mathrm{~S}$ & $<0.001$ \\
$\mathbf{0 . 0 3 1 2}$ & $5.32 \pm 0.58$ & $\mathrm{~S}$ & $<0.001$ \\
\hline
\end{tabular}

Table 2. Alignments of Neuraminidase $(n=10)$ Amino Acid Sequences From Influenza A(H1N1)pdm09 Viruses in Shiraz, Iran, in 2013

\begin{tabular}{lllllll}
\hline Accession Number & $\mathbf{1 3}$ & $\mathbf{1 0 6}$ & $\mathbf{2 4 1}$ & $\mathbf{2 4 8}$ & $\mathbf{2 7 5}$ & $\mathbf{3 6 9}$ \\
\hline FJ966084 & V & V & V & N & H & N \\
KJ755333 & G & I & - & D & K \\
KJ755334 & G & I & - & D & K \\
KJ755335 & G & I & I & K & K \\
KJ755336 & G & I & - & D & - & - \\
KJ789325 & G & I & I & - & - & - \\
KJ789326 & G & - & I & D & - & K \\
KJ789327 & G & I & I & D & K \\
\hline KJ789328 & - & I & I & D & - & K \\
\hline KJ789329 & - & I & I & D & - & - \\
\hline KJ789330 & - & I & - & - \\
\hline
\end{tabular}




\section{Discussion}

In April 2009, a new influenza A(H1N1) virus emerged in Mexico that quickly spread all over the world (20). This pandemic virus was first reported in June 2009 in Iran and became predominant during the 2009 influenza seasons (21). Increasing resistance of influenza A viruses to oseltamivir has recently been reported around the world (3). It is usually believed that oseltamivir resistant viruses emerge under the selective pressure of antiviral drug, but they are less pathogenic or transmissible than sensitive viruses (6). Resistance to oseltamivir in the $\mathrm{A}(\mathrm{H} 1 \mathrm{N1}) \mathrm{pdm09}$ viruses is associated with two mutations (H275Y and S247N). However, strains with H275Y mutation have the important advantage of rapid replication, which leads to a more widespread circulation (15). This study highlighted the prevalence of oseltamivir resistant A(H1N1)pdm09 viruses from 2012 to 2013 in Shiraz, Iran. This variant exhibited 5 mutations in NA gene, including V13G, V106I, V241I, N248D, and N369K.

Our study showed that V106I, V241I, N248D, and N369K mutations are most common substitutions in NA gene of $\mathrm{A}(\mathrm{H} 1 \mathrm{N1}) \mathrm{pdmog}$ virus. These 4 mutations in resistant and sensitive isolates occurred at the specific sites of NA in positions 106, 241, 248, and 369. Phylogenetic analysis showed that genetic features of $\mathrm{A}(\mathrm{H} 1 \mathrm{~N} 1)$ pdm09 variants isolated in Shiraz and other cities of Iran during 2012 and 2013 were also associated with $\mathrm{A}(\mathrm{H} 1 \mathrm{~N} 1)$ pdmo9 viruses isolated from our patients. On the other hand, the $\mathrm{A}(\mathrm{H} 1 \mathrm{~N} 1)$ pdmog viruses collected in this study revealed high homology to viruses of cluster B isolated in Shiraz in 2010.

The results showed that all of our isolates were sensitive to oseltamivir and phylogenetically close to the reference strain (A/California/4/2009). The results of the analysis of molecular test were consistent with those of phenotypic tests performed in cell cultures. Oseltamivir resistance to $\mathrm{A}(\mathrm{H} 1 \mathrm{~N} 1)$ pdm09 viruses with $\mathrm{H} 275 \mathrm{Y}$ mutation was detected in 4 patients in 2009 in Tehran, Iran. In this study, $\mathrm{A}(\mathrm{H} 1 \mathrm{N1}$ )pdmo9 viruses isolated from some patients, who did not consume oseltamivir and infected with these viruses were resistant to this drug. Thus, the results showed that the resistant virus could have emerged in the presence or absence of selective drug pressure (4). In 2009, another study performed in Shiraz, Iran showed no oseltamivir resistant $\mathrm{A}(\mathrm{H} 1 \mathrm{~N} 1)$ pdm09 viruses with $\mathrm{H} 275 \mathrm{Y}$ mutation (21). In a similar study conducted in Beijing, China, all of the $\mathrm{A}(\mathrm{H} 1 \mathrm{~N} 1)$ pdmo9 viruses isolated in 2012 2013 were sensitive to oseltamivir (22). However, studies conducted in the United States, Australia, Brazil, Italy, and the Asia-Pacific showed a significant increase in the oseltamivir resistant $\mathrm{A}(\mathrm{H} 1 \mathrm{~N} 1)$ pdmog viruses with $\mathrm{H} 275 \mathrm{Y}$ mutation $(2,15,17,18,23-25)$. Experiments conducted in cell cultures in Greece showed resistant variants with H275Y, to be the dominant population. This finding shows that the H275Y substitution does not compromise with the replication of $\mathrm{A}(\mathrm{H} 1 \mathrm{~N} 1) \mathrm{pdm} 09$ virus in vitro (8).

The analysis of oseltamivir resistance test results re- quires knowledge about the present laboratory procedures and their restrictions. Optimal testing procedures are different depending on the influenza virus type/subtype and drug testing. Therefore, commentary on antiviral sensitivity testing for clinical goals is complicated, and the results obtained may also vary with respect to different laboratories. The combination of applied and genotypic methods would be the most instructive and provide optimal testing method in connection with newly emerging oseltamivir resistant strain (9).

There is growing concern that $\mathrm{A}(\mathrm{H} 1 \mathrm{N1}) \mathrm{pdm} 09$ virus strains resistant to oseltamivir may appear and spread in a similar fashion to oseltamivir resistant $\mathrm{A}(\mathrm{H} 1 \mathrm{N1}$ ) seasonal virus (26). Our information about identification and transmissibility of oseltamivir resistant variant is still limited, and requires in vivo and in vitro studies. In addition, $\mathrm{A}(\mathrm{H} 1 \mathrm{N1}$ )pdmo9 virus must be carefully monitored to identify the emerging of drug resistant variants. Results of in vivo research in ferret and mice models in Lebanon revealed that oseltamivir resistant $\mathrm{A}(\mathrm{H} 1 \mathrm{N1}) \mathrm{pdm09}$ viruses are pathogenic and can be effectively transmitted via close contact, but not through respiratory droplets. Inefficient transfer of droplets of oseltamivir resistant A(H1N1) pdmo9 viruses could account for extremely low frequency of these viruses (2). In the present situation, the correct use of oseltamivir followed by careful surveillance is essential to identify drug-resistant A(H1N1)pdmo9.

\section{Acknowledgements}

The authors of this article would like to thank the Ethics Committee of SUMS for their approval of this project. In addition, we thank the Bacteriology and Virology Department staff for their assistance.

\section{Authors' Contributions}

Study concept: Afagh Moattari; Study design: Afagh Moattari and Mahmoud Shamsi Shahr abadi; Bench work: Nastaran Khodadad, Afagh Moattari, Forough Tavakoli, and Neda Pirbonyeh; Patients' diagnosis and selection: Mohammad Rahim Kadivar; Data analyses: Afagh Moattari, Jamal Sarvari, Amir Emami, and Nastaran Khodadad; Manuscript drafting: Nastaran Khodadad and Afagh Moattari; Critical revision of manuscript: Afagh Moattari.

\section{Funding/Support}

This study has been extracted from M.Sc. student thesis (Nastaran Khodadad) with the project No. 6544. This study was funded by Shiraz Influenza research center and deputy chancellor of Research both affiliated to Shiraz university of medical sciences.

\section{References}

1. Yavarian J, Azad TM, Zheng X, Gregory V, Lin YP, Hay A. Amantadine resistance in relation to the evolution of influenza $\mathrm{A}(\mathrm{H} 3 \mathrm{~N} 2)$ viruses in Iran. Antiviral Res. 2010;88(2):193-6.

2. Zaraket H, Kondo H, Tabet C, Hanna-Wakim R, Suzuki Y, Dbaibo 
GS, et al. Genetic diversity and antiviral drug resistance of pandemic H1N1 2009 in Lebanon. J Clin Virol. 2011;51(3):170-4.

3. Bai GR, Chittaganpitch M, Kanai Y, Li YG, Auwanit W, Ikuta K, et al. Amantadine- and oseltamivir-resistant variants of influenza A viruses in Thailand. Biochem Biophys Res Commun. 2009;390(3):897-901.

4. Moradi A, Nadji SA, Tabarsi P, Hashemian SM, Marjani M, Sigaroodi A, et al. Prevalence of Oseltamivir-Resistant 2009 H1N1 Influenza Virus among Patients with Pandemic 2009 H1N1 Influenza infection in NRITLD, Tehran, Iran. Tanaffos. 2011;10(1):8-11.

5. Monto AS. The role of antivirals in the control of influenza. Vaccine. 2003;21(16):1796-800.

6. Dapat C, Suzuki Y, Kon M, Tamura T, Saito R, Dapat IC, et al. Phylogenetic analysis of an off-seasonal influenza virus A (H3N2) in Niigata, Japan, 2010.Jpn J Infect Dis. 2011;64(3):237-41.

7. Pontoriero A, Baumeister E, Campos AM, Savy VL. Virological surveillance and antiviral resistance of human influenza virus in $\mathrm{Ar}$ gentina, 2005-2008. Rev Panam Salud Publica. 2011;30(6):634-40.

8. Gioula G, Melidou A, Exindari M, Papoutsi N, Chatzidimitriou D Dotis J, et al. Oseltamivir-resistant influenza A pandemic (H1N1) 2009 virus in Northern Greece. Hippokratia. 2011;15(3):272-4.

9. Nguyen HT, Trujillo AA, Sheu TG, Levine M, Mishin VP, Shaw M, et al. Analysis of influenza viruses from patients clinically suspected of infection with an oseltamivir resistant virus during the 2009 pandemic in the United States. Antiviral Res. 2012;93(3):381-6.

10. Takayama I, Nakauchi M, Fujisaki S, Odagiri T, Tashiro M, Kageyama T. Rapid detection of the S247N neuraminidase mutation in influenza A(H1N1)pdmo9 virus by one-step duplex RT-PCR assay.J Virol Methods. 2013;188(1-2):73-5.

11. Dapat IC, Dapat C, Baranovich T, Suzuki Y, Kondo H, Shobugawa Y, et al. Genetic characterization of human influenza viruses in the pandemic (2009-2010) and post-pandemic (2010-2011) periods in Japan. PLoS One. 2012;7(6):e36455.

12. Merten OW, Manuguerra JC, Hannoun C, van der Werf S. Production of influenza virus in serum-free mammalian cell cultures. Dev Biol Stand.1999;98:23-37.

13. Li Y, Zhang H, Xie M, Hu M, Ge S, Yang D, et al. Abundant expression of Dec1/stra13/sharp2 in colon carcinoma: its antagonizing role in serum deprivation-induced apoptosis and selective inhibition of procaspase activation. Biochem J. 2002;367(Pt 2):413-22.

14. Finney DJ. Statistical method in biological assay.London: Charles Griffin \& Co; 1952.

15. Jacks A, Ollgren J, Ziegler T, Lyytikainen O. Influenza-associated hospitalisations in Finland from 1996 to 2010: unexpected agespecific burden during the influenza A(H1N1)pdmo9 pandemic from 2009 to 2010. Euro Surveill. 2012;17(38)

16. Tamura K, Stecher G, Peterson D, Filipski A, Kumar S. MEGA6: Molecular Evolutionary Genetics Analysis version 6.0. Mol Biol Evol. 2013;30(12):2725-9.

17. Takashita E, Ejima M, Itoh R, Miura M, Ohnishi A, Nishimura $\mathrm{H}$ et al. A community cluster of influenza A(H1N1)pdmo9 virus exhibiting cross-resistance to oseltamivir and peramivir in Japan, November to December 2013. Euro Surveill. 2014;19(1):20666.

18. Souza TM, Resende PC, Fintelman-Rodrigues N, Gregianini TS, Ikuta N, Fernandes SB, et al. Detection of oseltamivir-resistant pandemic influenza A(H1N1)pdm2009 in Brazil: can community transmission be ruled out? PLoS One. 2013;8(11):e80081.

19. Ramirez-Gonzalez JE, Gonzalez-Duran E, Alcantara-Perez P, WongArambula C, Olivera-Diaz H, Cortez-Ortiz I, et al. Oseltamivirresistant pandemic (H1N1) 2009 virus, Mexico. Emerg Infect Dis. 2011;17(2):283-6.

20. Garten RJ, Davis CT, Russell CA, Shu B, Lindstrom S, Balish A, et al. Antigenic and genetic characteristics of swine-origin 2009 A(H1N1) influenza viruses circulating in humans. Science. 2009;325(5937):197-201.

21. Ziyaeyan M, Alborzi A, Jamalidoust M, Moeini M, Pouladfar GR, Pourabbas B, et al. Pandemic 2009 influenza A (H1N1) infection among 2009 Hajj Pilgrims from Southern Iran: a real-time RTPCR-based study. Influenza Other RespirViruses. 2012;6(6):e80-4.

22. Fang Q, Gao Y, Chen M, Guo X, Yang X, Yang X, et al. Molecular epidemiology and evolution of A(H1N1)pdmo9 and H3N2 virus during winter 2012-2013 in Beijing, China. Infect Genet Evol. 2014;26:228-40.

23. Hoang Vu MP, Nguyen CT, Nguyen le KH, Nguyen TK, Le QM. Oseltamivir resistance among influenza viruses: surveillance in northern Viet Nam, 2009-2012. Western Pac Surveill Response J. 2013;4(2):25-9.

24. Arvia R, Corcioli F, Simi L, Orlando C, De Santis R, Facchini M, et al. Monitoring the susceptibility to oseltamivir of Influenza A(H1N1) 2009 virus by nested-PCR and pyrosequencing during the pandemic and in the season 2010-2011. J Virol Methods. 2012;184(1-2):113-6.

25. Tarbet EB, Vollmer AH, Hurst BL, Barnard DL, Furuta Y, Smee DF. In vitro activity of favipiravir and neuraminidase inhibitor combinations against oseltamivir-sensitive and oseltamivir-resistant pandemic influenza A (H1N1) virus. Arch Virol. 2014;159(6):1279-91.

26. Oh DY, Hurt AC. A Review of the Antiviral Susceptibility of Human and Avian Influenza Viruses over the Last Decade. Scientifica (Cairo). 2014;2014:430629. 